4.

TRANS · núm. I $5 \cdot 201 \mathrm{I}$

NOTA $\cdot 211-218$
Tradicionalmente, la determinación de los parámetros de presentación de los subtítulos ha quedado establecida a través de las directrices emitidas por las productoras 0 empresas de subtitulado, los organismos reguladores o los expertos en investigación sobre traducción audiovisual. Las nuevas tecnologias permiten un análisis independiente y exacto basado en el producto final que el consumidor recibe en formato digital. Con este análisis se pueden definir las tendencias reales que, con el paso del tiempo, ha seguido la industria de la subtitulación.

PALABRAS CLAVE: traducción audiovisual, subtítulos, subtitulación, velocidad de lectura.

\title{
Análisis diacrónico de la velocidad de presentación de subtítulos para DVD
}

\section{J. DAVId GonZÁLEZ-IgleSIAS GONZÁLEZ}

Universidad de Salamanca

\section{Diachronic Analysis of Reading Speed in Subtitles for DVD}

Traditionally, presentation parameters for subtitles have been determined by the guidelines issued by production or subtitling companies, regulatory bodies or research experts in the field of audiovisual translation. New technologies allow for an independent and accurate analysis based on the final product that the consumer receives in a digital format. From this analysis we can define the actual trends that the subtitling industry has been applying over time.

KEY WORDS: audiovisual translation, subtitles, subtitling, reading speed. 


\section{INTRODUCCIÓN}

Desde principios de la década de los 9o, la 212 investigación en el campo de la traducción audiovisual (TAV) se ha propuesto como uno de sus múltiples objetivos fijar unos parámetros estables y aceptables para los subtítulos que se emiten en proyecciones de cine y televisión. A partir de las primeras propuestas de Ivarsson en 1992 (complementadas posteriormente junto a Carroll en r998), se llegó con Dries en r995 y Karamitroglou en 1998 a unas primeras iniciativas para la subtitulación en todo el ámbito europeo. Desde ese momento, los teóricos y profesionales de la TAV en nuestro país han revisado constantemente estos parámetros con el fin de ajustarse en la medida de lo posible a las exigencias del mercado. No obstante, el grueso de la investigación hasta la fecha se ha basado en las directrices emitidas por las productoras, como es el caso de la 2oth Century Fox, cuyas normas desgrana Mayoral (200I), o por los organismos reguladores correspondientes (Dries, I995) o, en algunos casos, en la propia experiencia profesional de los autores (Castro Roig, 20orb). De este modo, las observaciones no se han fundamentado nunca en un análisis cuantitativo real de los subtítulos tal y como aparecen finalmente en pantalla en el producto acabado, por lo que no se ha podido determinar con exactitud en qué medida las emisiones que consume el público se ajustan a los dictámenes académicos y/o del mundo profesional.

Por nuestra parte, la motivación que ha dirigido nuestros pasos es la que se refleja en las recomendaciones de Castro Roig (2001a: I38) y Mayoral (2005: 7), que hablan de la importancia de incorporar las nuevas tecnologías en la investigación sobre traducción audiovisual, y de investigar sobre las novedades tecnológicas en el sector. En este sentido, es innegable que la proliferación masiva del DVD como elemento habitual integrado en los equipos informáticos ha supuesto un salto cualitativo fundamental en este campo. Las innovaciones que presenta este soporte resultan decisivas en lo referente a la experiencia del espectador, que ahora tiene acceso a la combinación lingüística que prefiera (Díaz-Cintas, 2003a: I98; 2005: I3), así como a la modalidad de traducción que elija (doblaje o subtitulado). Además, a través de los subtítulos y de los redoblajes, el espectador tiene a su alcance un uso actualizado del lenguaje (O'Hagan, 2007: I65). No obstante, quizá los principales cambios que traen consigo estas nuevas aportaciones sean aquellos que afectan a la metodología investigadora. Como ejemplo, baste señalar la observación de Kayahara (2005), quien destaca que la llegada del DVD supone que los estudiosos e investigadores de la traducción audiovisual ya no necesitan dos cintas de vídeo para trabajar con los subtítulos y el doblaje a la vez, sino que lo tienen todo en uno.

Otra virtud de la aplicación de nuevas tecnologías en la investigación sobre traducción audiovisual, derivada del uso del DVD pero independiente de este, es la digitalización total del proceso. Ahora es posible llevar a cabo todas las etapas de obtención del corpus sin apartarse del ordenador, tal como ya apuntaba en 2005 el grupo de investigación Gent-Tau (De Yzaguirre et al., 2005), que contaba con todo un corpus de guiones de documentales (originales y traducidos) en formato digital para trabajar con ellos. De igual modo, nosotros hemos podido manejar archivos informatizados, lo cual supone sin duda alguna un ahorro importante de tiempo y energía.

El último aspecto importante que hemos tenido presente a lo largo del trabajo de investigación es el que recalca Toda (2003: 282): 
Para ese tipo de estudios, y para intentar comprender por qué se escogió una forma [de expresión lingüística] y no otra que nos parece más adecuada, es absolutamente necesario trabajar con criterios técnicos, es decir, medir la duración del parlamento para poder ver «de cuánto disponían» el traductor (y el ajustador) para meter su texto. [...] Creo que eso es una buena formación no sólo para el ejercicio profesional como traductores especializados, sino para la investigación de carácter académico, que se podrá hacer de forma más rigurosa si tenemos presente ese encorsetamiento.

Así, ya desde el planteamiento inicial de este estudio, hemos pretendido contar con los parámetros técnicos como elemento fundamental a la hora de analizar cualquier traducción de nuestro corpus. Entendemos que son precisamente estos factores los que particularizan el género audiovisual, y que es esta la única forma de que se pueda avanzar en el conocimiento específico de esta especialidad. Coincidimos además con Toury (1995) en que para llevar a cabo el análisis de las normas, únicamente podemos observar sus manifestaciones, es decir, el producto original y el traducido, en tanto que fuentes textuales.

\section{OBJETIVOS}

El objetivo de nuestro estudio es determinar si existe una evolución a lo largo del tiempo en la cantidad de «subtítulos rápidos» y la velocidad de dichos subtítulos en los productos audiovisuales. Para ello, partimos de la hipótesis de que la velocidad de lectura de subtítulos del público aumenta con el tiempo, y de igual modo aumenta dicha velocidad en los productos que este consume. Así, nos encontramos ante un microestudio descriptivo que pretende analizar la evolución de la velocidad de lectura. Su alcance topográfico se limita a España y su ámbito temporal, diacrónico, abarca el período comprendido entre los años 2004 y 2010, ambos inclusive, que es aquel en el que se ha creado nuestro corpus de estudio.

Como corpus de estudio utilizamos la serie de televisión Perdidos (Lost, en su versión original, creada por Jeffrey Lieber, J. J. Abrams y Damon Lindelof y emitida en el periodo anteriormente mencionado), con las cinco temporadas que se han editado hasta la fecha en DVD en nuestro país, que constituyen nuestras fuentes primarias. Además, nos serviremos para las fuentes secundarias de obras de expertos nacionales e internacionales en el campo de la traducción audiovisual que han intentado ofrecer una perspectiva general acerca de los parámetros técnicos de presentación de los subtítulos. Cabe añadir que este estudio es de tipo cuantitativo, puesto que nuestra atención se centra en criterios numéricos, y no en el contenido léxico-semántico de los subtítulos. Sin embargo, su finalidad última es que esto nos permita tomar decisiones más fundamentadas acerca de las formas lingüísticas que se eligen. Además, su finalidad última no es teórica sino aplicada, puesto que la constatación de los fenómenos que analizamos podría permitirnos elaborar nuevas directrices para la industria de la traducción audiovisual.

\section{DELIMITACIÓN DE LAS VARIABLES}

Nuestro estudio gira en torno a dos variables dependientes y distintas. Por una parte, la cantidad de «subtítulos rápidos», definidos como aquellos subtítulos que exijan por parte del espectador una velocidad de lectura superior a $\mathrm{I} 7$ caracteres por segundo, y, por otra parte, la velocidad promediada de los mismos con respecto a una única variable independiente: el tiempo. 


\section{DISEÑO DE LA RECOGIDA DE DATOS}

\section{Descripción general}

214
Para nuestro estudio obtendremos los archivos correspondientes a los subtítulos en castellano de cinco capítulos que se corresponden con cada una de las cinco temporadas de la serie de televisión Perdidos en DVD. A partir de dichos archivos, extraeremos los tiempos de entrada y salida de cada uno de los subtítulos de los episodios y la longitud en caracteres de los mismos, con el objetivo de hallar la velocidad de lectura correspondiente a cada uno de ellos.

Como corpus de análisis, se han tomado los siguientes capítulos, elegidos de manera aleatoria (tabla I).

\section{Modelo de herramienta utilizada}

Para obtener los archivos de subtítulos, ha sido necesario emplear un programa informático de extracción de la capa de subtítulos (el llamado subpack) de los DVD originales, que contiene las pistas con los distintos idiomas. En nuestro caso, hemos utilizado el programa VobSub Ripper ${ }^{\mathrm{I}}$. A continuación, ha sido necesario hacer uso de una aplicación que extraiga el texto a partir de los archivos en los que se contiene esa capa de subtítulos. Para el presente trabajo hemos utilizado la aplicación Subtitle Edit ${ }^{2}$, que se basa en un software de reconocimiento óptico de caracteres (OCR, en sus siglas en inglés), según el cual el usuario asigna un carácter a cada una de las matrices de texto del capítulo. Este proceso requiere aproximadamente una hora por capítulo, y permite obtener las listas en formato .srt, que se puede editar cómodamente en el procesador de

\footnotetext{
I Disponible para su descarga gratuita en www.freecodecs.com/download/VobSub_Ripper.htm.

2 Disponible para su descarga gratuita en www.nikse. $\mathrm{dk} / \mathrm{se}$.
}

\section{TABLA I}

Episodio elegido para cada temporada

Temporada Fecha depublicación en DVD Episodio

\begin{tabular}{rlr}
\hline I & I4 diciembre 2005 & 9 \\
2 & 24 octubre 2006 & I6 \\
3 & Io octubre 2007 & 7 \\
4 & 26 noviembre 2008 & 8 \\
5 & 3 febrero 20I0 & I4 \\
\hline
\end{tabular}

textos más básico incluido en cualquier sistema operativo. Los archivos, además, presentan los subtítulos numerados de forma correlativa e incluyen los códigos de tiempo de entrada y salida de cada uno de ellos, expresados en horas, minutos, segundos y milésimas; lo cual, como veremos más adelante, será también de gran utilidad.

Una vez se han recopilado los elementos necesarios, y después de haberlos convertido a formatos digitales que puedan utilizarse con comodidad, pasamos a la segunda fase: el análisis del corpus. A lo largo de esta etapa, hemos llevado a cabo operaciones de diversa índole para obtener los datos necesarios en nuestro estudio. Sin embargo, quizá sea conveniente que nos detengamos en este punto para ofrecer la descripción de tres elementos básicos en el análisis de un subtítulo, y que nos serán útiles para comprender los procesos posteriores:

Longitud Se define la longitud del subtítulo como el número total de caracteres de los que consta (incluyendo espacios en blanco y signos de puntuación). En los casos de subtítulos de dos líneas, el paso de carro entre la primera y la segunda línea no cuenta como un carácter más.

Duración La duración del subtítulo es el tiempo que este permanece en pantalla. Se obtiene como resta entre el Tiempo de salida y 
el Tiempo de entrada de un mismo subtítulo y se mide en segundos y milésimas de segundo.

Caracteres por segundo (CPS) Se definen los caracteres por segundo como el cociente entre la longitud del subtítulo y su duración. Este parámetro corresponde a la velocidad necesaria para leerlo.

Cabe realizar una aclaración con respecto al parámetro de velocidad de lectura, para el que se ha elegido la unidad de caracteres por segundo, frente a otras que también se aplican en el sector, como la de palabras por minuto (WPM), en sus siglas en inglés. Se ha optado por el primero ante el segundo por dos motivos. Por una parte, la bibliografía especializada en castellano al respecto hace referencia principalmente a los CPS como medida de velocidad. Por otra parte, al tratarse de un estudio cuantitativo que no entra en la recepción de los subtítulos, entendemos que el valor en CPS resulta más preciso que el de WPM, que entiende a la palabra normalmente, pero no en todos los casos, como una unidad ideal de 5 caracteres además del espacio que la separa de la siguiente.

Así pues, con el objetivo de establecer el número de subtítulos que se presentan en cada uno de los capítulos, nos hemos servido de los archivos en formato .srt que obtuvimos en la fase anterior, puesto que en ellos se numera cada uno de los textos que aparecen en pantalla. A continuación, tratamos de calcular las velocidades de lectura particulares para cada uno de los subtítulos. Para ello, comenzamos por trasladar los textos de su formato original (.srt) al formato .doc, de forma que se pudieran editar en el procesador de textos Microsoft Word. Una vez realizado el cambio, se obtuvieron cinco documentos (uno por cada temporada, en castellano) que incluían los tiempos de entrada y salida para cada subtítulo, así como el texto de cada uno de ellos.
El proceso aplicado a cada uno de dichos documentos fue el siguiente: mediante el uso de las funciones básicas de buscar y reemplazar, vamos «limpiando» la lista, con el objetivo de dejar únicamente los elementos que nos interesan. Así, separamos por parejas los tiempos de entrada y salida de los subtítulos, por un lado, y los propios textos, por otro. Seguidamente, tras pasar las listas de tiempos a una hoja de cálculo (nosotros hemos utilizado la aplicación Microsoft Excel), obtuvimos la duración asignada a cada subtítulo mediante la resta del segundo valor de cada par del primero (el tiempo de salida menos el de entrada da como resultado el tiempo que el subtítulo permanece en pantalla). A continuación, redujimos los textos de cada subtítulo a una sola línea (en el caso de aquellos que aparecían en dos) para poderlos tratar como cadenas de caracteres, las trasladamos a la misma hoja de cálculo, y hallamos la longitud de cada una de ellas. Una vez obtenidas las extensiones de los subtítulos y los tiempos asignados a cada uno de ellos, resulta sencillo establecer qué velocidad de lectura (en caracteres por segundo) se exige del espectador en cada subtítulo. La iteración de este sistema para cada uno de los documentos nos permitió reunir en un solo archivo de Excel todos los subtítulos de nuestro estudio, junto con sus tiempos de entrada y salida, sus duraciones y la velocidad de lectura requerida para cada uno de ellos.

A partir de las indicaciones de distintos autores, como Mayoral (1993), Castro Roig (200Ib) o Díaz-Cintas (2003b), hemos establecido una velocidad máxima de lectura en i6 caracteres por segundo. Así, todos los subtítulos con una velocidad de lectura superior pasarán a considerarse «subtítulos rápidos» que exceden los parámetros óptimos de presentación. No 
obstante, tal y como aconsejan los autores antes citados, se debería permitir un cierto «margen de tolerancia» (Mayoral, r993: 57) de en torno al 216
5-10\% por encima de esa cifra, por lo que hemos seleccionado todos los segmentos de texto que requieran una velocidad superior a I7 caracteres por segundo.

El siguiente paso fue determinar cuántos de los subtítulos exigían una velocidad de lectura que se salía de los niveles aceptados. Nuevamente, la hoja de cálculo permite obtener esos datos de manera prácticamente instantánea, mediante las fórmulas correspondientes. Así, establecimos rápidamente el número absoluto y el porcentaje de estos subtítulos. Por último, se ha calculado el promedio de duración de los mismos.

Con estos factores en cuenta, veamos pues la tabla 2 en que se recogen los datos previamente mencionados.

\section{ANÁLISIS DE LOS DATOS OBTENIDOS}

A la luz de estos datos, podemos observar que la cantidad de subtítulos rápidos, que van aumentando a medida que avanzan las temporadas. De igual modo, el porcentaje de dichos subtítulos dentro del total se incrementa sensiblemente. Por último, podemos observar cómo la velocidad promediada de los subtítulos rápidos va en aumento hasta subir, en casi dos puntos, a lo largo de un período de seis años, el promedio de velocidad de lectura de aquellos subtítulos que se presentan a más de 17 caracteres por segundo. No podemos olvidar, no obstante, que se trata de un valor promediado, por lo que dentro de cada uno de los capítulos podemos encontrar subtítulos que sobrepasan los 25 CPS (episodio $5 \times$ I4; subtítulo I34: $27,752 \mathrm{CPS}$ ), los $30 \mathrm{CPS}$ (episodio $3 \times$ o7; subtítulo $265: 31,765 \mathrm{CPS}$ ), o que se acercan incluso a los $40 \mathrm{CPS}$ (episodio $3 \times 07$; subtítulo 262:39,289 CPS).

\section{CONCLUSIONES}

En vista de los resultados obtenidos, y conscientes de que nos encontramos ante unos datos provisionales, en tanto en cuanto el análisis sólo se ha efectuado con un episodio de cada una de las temporadas, podemos elaborar unas primeras conclusiones con respecto a los parámetros técnicos de presentación de los subtítulos.

-A medida que avanza el tiempo, el porcentaje de subtítulos que supera la velocidad de lectura de 17 caracteres por segundo aumenta hasta duplicarse en un espacio de cuatro años, como resultado probable de una menor condensación en los subtítulos.

-Dentro de ese mismo espacio de tiempo, la

TABLA 2. Relación de porcentaje de subtítulos «rápidos» y velocidad promedio de lectura

\begin{tabular}{|c|c|c|c|c|}
\hline Episodio & $\begin{array}{l}\text { Cantidad } \\
\text { de subtitulos }\end{array}$ & $\begin{array}{l}\text { Cantidad de subtitulos } \\
>I 7 \text { CPS }\end{array}$ & $\begin{array}{l}\text { \% de subtitulos } \\
>\text { I } 7 \text { CPS }\end{array}$ & $\begin{array}{l}\text { Velocidad de lectura } \\
\text { promedio de subtitulos }>\text { I7 CPS }\end{array}$ \\
\hline IxO9 & 505 & $5^{\mathrm{I}}$ & IO,IO & $\mathrm{I} 8,00$ \\
\hline $2 \mathrm{xI} 6$ & 490 & 55 & $\mathrm{II}, 22$ & $\mathrm{I} 8,54$ \\
\hline $3 \times 07$ & 450 & 64 & I 4,22 & I8,58 \\
\hline $4 \times 08$ & 457 & 86 & 18,82 & 19,28 \\
\hline $5 \times 14$ & 503 & IO7 & 21,27 & 19,85 \\
\hline
\end{tabular}


densidad de dichos subtítulos aumenta ligera pero invariablemente hacia una velocidad de lectura media que se sitúa cerca de los 20 caracteres por segundo.

Por último, podríamos añadir que esta tendencia alcista, que cuenta, en principio, con la aprobación del público, puesto que no se han producido muestras de rechazo ante el aumento de las velocidades, puede verse justificada por el hecho de que el espectador medio de series de televisión en DVD haya adquirido ya una costumbre afianzada en la lectura de subtítulos. Así, sería razonable esperar que esta tendencia se generalice y llegue a un nuevo punto de estabilización que nos permita redefinir los parámetros de presentación de subtítulos en los productos destinados al DVD y/o a la televisión digital terrestre. A este último respecto es importante destacar que, si bien hasta ahora la gran mayoría de series de televisión no se han podido disfrutar con subtítulos en las cadenas generalistas en abierto, el fin de la televisión analógica en España amplía potencialmente el mercado de consumo de subtítulos a la totalidad de la población.

RECIBIDA EN ABRIL DE 2010 ACEPTADA EN JULIO DE 2010 VERSIÓN FINAL DE OCTUBRE DE 2010

\section{BIBLIOGRAFÍA}

Castro Roig, X. (20ora). "Cuestiones sobre la norma culta y los criterios de calidad para la traducción de doblaje y subtitulación en España», en Chaume, Varela, F. y Agost, R. (eds.). La traducción en los medios audiovisuales, Castelló: Publicacions de la Universitat Jaume I, pp. I35-I4O.

- (200Ib). «El traductor de películas», en Duro, M. (coord.). La traducción para el doblaje y la subtitulación, Madrid: Cátedra, pp. 267-298.

De Yzaguirre, L; García, Y.; Matamala, A.;
Ribas, M. (2005). «Tratamiento informático de un corpus de guiones y traducciones de textos audiovisuales: Implicaciones en traductología», en Zabalbeascoa, P., Santamaria, L. y ChauME VARELA, F. (eds.). La traducción audiovisual: Investigación, enseñanza y profesión, Granada: Comares, pp. IO7-II4.

Díaz Cintas, J. (2003a). «Audiovisual Translation in the Third Millenium», en Anderman, G. y Rogers, M. (eds.). Translation Today: Trends and Perspectives, Clevedon (England): Multilingual Matters, pp. 192-204.

- (2003b). Teoría y práctica de la subtitulación. InglésEspañol, Barcelona: Ariel.

- (2005). «Nuevos retos y desarrollos en el mundo de la subtitulación». Puentes, 6, pp. 13-20.

Dries, J. (1995). Dubbing and subtitling: guidelines for production and distribution, Düsseldorf: European Institute for the Media.

Ivarsson, J. (1992). Subtitling for the media, Stockholm: Transedit.

- y Carroll, M. (1998). Subtitling, Simrisham: TransEdit HB.

Karamitroglou, F. (I998). "A proposed set of subtitling standards in Europe». Translation Journal, 2/2. En línea: http://accurapid.com/ journal/o4stndrd.htm. Última consulta: julio de 2010.

Kayahara, M. (2005). «The digital revolution: DVD Technology and the Possibilites for Audiovisual Translation Studies». The Journal of Specialised Translation, 3, pp. 64-74. En línea: http://www. jostrans.org/issueo3/art_kayahara.php. Última consulta: julio de 2010 .

Mayoral, R. (I993). «La traducción cinematográfica: el subtitulado». Sendebar, 4, pp. 45-68.

- (200I). "El espectador y la traducción audiovisual», en Chaume, F. y Agost, R. (eds.). La traducción en los medios audiovisuales, Castelló: Publicacions de la Universitat Jaume I, pp. 33-46.

- (2005). «Reflexiones sobre la investigación en traducción audiovisual», en ZabALBEASCOA, P., Santamaria, L. y Chaume Varela, F. (eds.). La traducción audiovisual: Investigación, enseñanza y profesión, Granada: Comares, pp. 3-8.

O'Hagan, M. (2007). «Impact of DVD on Translation: Language Options as an Essential Add-On Feature». Convergence: The international Journal 
of Research into Nerw Media Technologies, I3/2, pp. I57-I68.

TodA, F. (2003). «La investigación en traducción audiovisual en el Tercer Ciclo: algunas consideraciones», en García Peinado, M.A. y Ortega
Arjonilla. E. (dirección). Panorama actual de la investigación en traducción e interpretación (vol. 2), Granada: Atrio, vol. II, pp. 269-285.

Toury, G. (1995): Descriptive Translation Studies and Beyond, Amsterdam/Filadelfia: John Benjamins. 